\title{
Prevalence of Malaria in a Study Population in Maiduguri, Nigeria, Symptomatic Manifestations and Public Health Implications
}

\author{
${ }^{1}$ DAVID J, ${ }^{1}$ ANASO EC, ${ }^{2 \star}$ OZURUMBA LN
}

\author{
${ }^{1}$ Department of Biological Sciences, University of Maiduguri, Maiduguri, Borno State, Nigeria. \\ ${ }^{2}$ School of Public Health, Walden University, Minnesota MN, United States of America; Previously \\ Department of Biological Sciences, University of Maiduguri, Nigeria.
}

\section{ARTICLE INFO}

Article No.: 092416145

Type: Research

DOI: 10.15580/GJBS.2016.5.092416145

Submitted: $24 / 09 / 2016$

Accepted: 05/10/2016

Published: 30/11/2016

*Corresponding Author

Ozurumba LN

E-mail: leo.ozurumba@waldenu.edu Phone: +2347036018070

\section{Keywords:}

Plasmodium, infection, symptoms, pathology, seasons, prevalence, public, health

\section{ABSTRACT}

A Questionnaire based study was conducted at the University of Maiduguri Teaching Hospital (UMTH), Maiduguri, Borno State, Nigeria on the nature of symptoms observed in patients who were presumptively treated for malaria based on observed symptoms from which analysis was done on the occurrences of each of these symptoms. Then, the prevalence of malaria infection was investigated through data collection at the same hospital. These sets of data collected was centred on patients who attended the hospital (UMTH) between January, 2011 to December, 2011. The age of the patients ranged from 1 to 90 years.

Results from questionnaire completed by the medical personnel at the hospital indicated that vomiting had the highest occurrence at $100 \%$. Other symptoms such as fever, headache, joint pain and nausea ranked next to vomiting in occurrence with figures of between $64.7 \%$ and $94.1 \%$, while symptoms such as jaundice, loss of consciousness, weight loss, arthralfia, bleeding, pyrexia, cough, backache, and reduced uterine output were the least in occurrence at between $5.9 \%$ and $11.8 \%$. Apparently, symptoms such as Bleeding, jaundice, reduced uterine output are associated with pathologies of malaria parasites which appear to be flourishing in proliferation and are ferried to other parts of the body by the blood, where they are establishing new sites of more pronounced damages to host tissues (blood cells and other systemic related cells) associated with the related pathologies and emergence of these symptoms.

It is suggested that treatment for patients should take into cognizance the most occurring symptoms, the reasons for this trend and incorporation certain drugs or slight adjustments of spectrum of drugs to accommodate effective management of this issue; while the skewed peak transmission season seem to indicate increased level malaria control steps during these period.

A total of 271 of these individuals were examined by laboratory technicians through a method that involved blood smear making, appropriate staining of slides and examination of the blood smears for the presence of malaria parasites. Male patients recorded malaria parasite prevalence of $43.9 \%$ while for female patients it was $56.1 \%$. This \%prevalence difference based on gender does not appear significant.

Peak malaria prevalence was recorded in the month of September and followed by June (15.58\% and $14.02 \%$ respectively) while the value was least for March followed by November ( $2.21 \%$ and $3.69 \%$ respectively). This is indicative of skewness of most of the peak related values in the rainy season compared to the dry season. The sahel savannah related long stretch of dry climatic conditions of this area which provide breeding conditions during the rainy seasons is likely to be one of the contributors to this observation, alongside other human malariogenic and environmental factors which favour increased breeding of the mosquito vector. 


\section{INTRODUCTION}

Malaria is a protozoan apicomplexan mosquito-borne parasitic disease of the genus Plasmodium (a Eukaryotic Protist) occuring in humans and other animals. Severe disease is largely caused by Plasmodium falciparum, while the disease caused by Plasmodium vivax, Plasmodium ovale and Plasmodium malariae are generally milder and rarely fatal. Plasmodium knowlesi a zoonotic malaria parasite that causes malaria in macaques but can also infect humans. These species of Plasmodium differ morphologically, immunologically, geographically, in distribution, in relapse pattern, in incubation time and in drug response (Cheesebrough, 1998, AMCA, 2014).

There are approximately 156 named species of Plasmodium which infect various species of vertebrates. Four are mostly known to infect humans: $P$. falciparum, $P$. vivax, P.malariae, and $P$. ovale. It is one of the very important tropical diseases in terms so morbidity and mortality. Worldwide, some two billion individuals are at risk; and 100 million develop over clinical disease. Nearly $85 \%$ of the cases and $90 \%$ of carriers (many asymptomatic) are found in tropical Africa. The incidence of malaria is increasing due to resistance of vectors to insecticides and drug resistant parasites. Several centuries after the discovery of malaria, it remains a devastating human infection, totaling between 300 to 500 million clinical cases and more than 1 million deaths every year. The complexity of the disease vector (the female anopheles mosquito) is only exceeded by the complex life cycle of the parasite (AMCA, 2014).

Of the four species that infect humans, $P$. vivax and $P$. falciparum account for $95 \%$ of infections. $P$. vivax has widest distribution, extending throughout the tropics, subtropics, and temperate zones. $P$. falciparum is generally confined to the tropics, $P$. malariae is sporadically distributed, and $P$. ovale is confined mainly to Central West Africa and some south pacific islands. In India, $P$. vivax and $P$. falciparum are very common, a few cases of $P$. malariae and $P$. ovale have also been reported. (Arora and Arora, 2010).

Mosquito borne illnesses include malaria, West

Nile Virus (WNV), Elephantiasis, Eastern Equine Encephalitis (EEE), Dengue fever, Dog Heartworm, Chikungunya and yellow fever. Over 1million people worldwide die from mosquito borne diseases very year (AMCA, 2014). In Nigeria, incidence of malaria also varies with weather, which influences the ability of the main carrier of malaria parasites, Anopheline mosquitoes, to survive or otherwise. Country specific evidence shows that Nigeria has the largest population at risk of malaria in Africa and therefore most vulnerable to the risk of missing millennium development goal target. Malaria is a major health problem in Nigeria with stable transmission throughout the country. It accounts for about 60 percent of out-patient consultation, about 30percent of hospital admission, and also prime among the top three causes of death in Nigeria. Approximately
$50 \%$ of Nigeria population experience at least one episode per year (LSM, 2016). While many species are native to tropical and sub-tropical regions, some such as Aedes spp have successfully adapted to cooler regions. In the warm and humid tropical regions, they are active in the entire year long, however, in temperate regions, they hibernate over winter. Eggs from strains in the temperate zones are more tolerable to the cold than ones from warmer regions (Hanson and Craig, 1995). They can even tolerate snow and temperatures under freezing. In addition, adults can survive throughout winter in suitable microhabitat (Romi et al, 2006).

Signs and symptoms of malaria include headache, fever, fatigue, muscular pain, back pain, vomiting, nausea, spleen enlargement, dry cough, sweating, skin chills, shivery, arthralgia (joint pain), anemia, haemoglobinuria, retinal damage and convulsion. Symptoms usually appear between ten and fifteen days after mosquito bites. An individual infected with Plasmodium falciparum, if not treated immediately may have start having complications which may result in experiencing more devastating symptoms like kidney failure, seizures, mental confusion, comma and death.

The above symptoms are grouped into three stages namely cold stage, hot stage and sweat stage. For instance, the cold stage begins with shaking chills usually at mid-day between 11 am to 12 noon and this last from 15 minutes to an hour; while the following hot stage is characterized by high fever even reach above $106^{\circ} \mathrm{F}\left(41^{\circ} \mathrm{C}\right)$. The fever can last for two to six hours.

Pregnant women, children below 5years and non-immuned individuals are at greater risk of infection and death from malaria (Gellert et al, 1998; Noland et al, 2014; WHO, 2016a and 2016b).

Malaria parasites exhibit a complex life cycle involving alternating cycles of a phase of asexual division (schizogony) occurring in man (intermediate host) and a phase of sexual division (sporogony) occurring in female Anopheles mosquito (definite host). Therefore, malaria parasites exhibit alternation of generations and alternation of hosts. The sporozoites are the infective form of the parasite and are present in the salivary glands of female Anopheles mosquito. Man gets infected by the bite of infected mosquito. It usually bites at night or during the twilight hours, either right after sunset or before sunrise.

The prevalence of malaria varies from region to region on the globe, being notably higher in the tropical countries than in the cold climate western world. This is prominently because the climate in the tropics supports breeding of the vector Anopheles mosquito which transmits the disease from person to person, alongside other factors (such as habits, some genetic factors and the environment, which influence the level of malaria prevalence (Ukoli, 1990; Ozurumba et al, 2016; Umaru and Uyaibasi, 2015).

Varying figures of malaria prevalence has been recorded in various parts of Nigeria (a tropical country) $35.7 \%$ in Kaduna (Umaru and Uyabaisi,2015), and 36- 
$6 \%$ in Plateau, and higher values ranging from 40 to $70 \%$ in some other areas (Udom et al, 2013).

\section{SIGNIFICANCE OF THIS STUDY}

Malaria is ranked the third among major infectious diseases threatening Africa after Pneumococca acute respiratory infection (3.5\%) and tuberculosis (2.8\%). Malaria occurs throughout Nigeria in all the country's ecological zones. Statistics had it that more than 64 millions Nigerians are reportedly attacked every year by malaria parasites transmitted by mosquitoes, while $25 \%$ of children are killed by the disease. Records showed that about 300,000 Nigerians mostly children under five years of age are killed every year by malaria (Gellert et al, 1998; Noland et al, 2014; LSM, 2016; WHO, 2016a and 2016b).

Malaria constitutes a menace, an enormous danger to the physical and social economic development of the nation. Malaria impedes the flow of trade, foreign investments and commerce, thereby affecting the country as a whole when its prevalence and burden is fairly high or high. It hinders a child's physical and cognitive development. These have public health implications. The effect of malaria can result into absenteeism and low performance among school children who are being groomed to later in future make up country's labour force or human resources which has inkling on the nature of economic growth of such a nation.

This study presents some leads from our findings in the area of manifested spectrum and occurrence frequencies of specific symptoms of malaria among the study population in relation to the pathobiology of malaria infection, albeit aspects of the pathogenesis of the disease. Based on these findings and discussions on it, some useful hints and links useful in the management of the diseases by medical personnel are proffered.

\section{The specific objectives of this study are to:}

* Determine the most prominent malaria symptom in the study population and symptoms which are the closest in occurrence level to the highest.

* Have a clue as to the spectrum or profile of entire pool of manifested symptoms.

* Evaluate the gender based prevalence levels (sex of patients based) of malaria of the individuals and inspect for margin of difference between both sexes.

* Determine the monthly based prevalence of infection in the study population in the period of study between January and December, 2011; and the pattern of this monthly prevalence distribution.

\section{MATERIALS AND METHODS}

\section{Study Area}

Maiduguri the capital of Borno state is located at longitude $11^{\circ} \mathrm{E}$ and latitude $3^{\circ} \mathrm{N}$ to the Northern part of Nigeria. Borno state is bordered by the neighboring counties of Cameroon and Chad on the east.

The hot climate has mean temperature of $38^{\circ} \mathrm{C}$, the hottest months are March, April and May with temperature ranging from $30^{\circ} \mathrm{C}$ to $40^{\circ} \mathrm{C}$. The rainy season starts from June and last for about four (4) months. Maiduguri is the site where University Teaching Hospital is located.

This study engaged the collection of data though medical doctors and nurses who completed the questionnaire on symptoms recorded on each of the patients who were symptomatically diagnosed for malaria and scheduled to undergo the treatment course for malaria at the University of Maiduguri Teaching Hospital, Maiduguri, Borno State, between January, 2011 to December, 2011. The age of the patients ranged from 1 to 90 years.

Also, data was retrieved from results of laboratory diagnostic tests conducted on patients who attended the hospital (UMTH) from January, 2011 to December, 2011. A total of 271 of these individuals were examined by laboratory technicians through a method that involved blood collection by finger pricking on the patients, blood smear making on slides, appropriate staining of slides using the Leishman's staining method and examination of the blood smears for the presence of malaria parasites.

\section{Analysis of Results}

The results were analyzed using Micosoft Excel with functions for mean and graphical statistical analytic functions engaged from this package.

\section{RESULTS}

From questionnaires filled by Doctors and Nurses at University of Maiduguri Teaching Hospital, our analysis produced the following results presented as Tables and Figures. 
Table 1: Symptoms of Malaria recorded in the study population

\begin{tabular}{|c|c|c|c|c|c|c|c|}
\hline \multirow{2}{*}{$\frac{S N}{1}$} & \multirow{2}{*}{$\frac{\text { symptoms }}{\text { Headache }}$} & \multicolumn{2}{|c|}{ Number of times appearedirom questionnaire } & \multicolumn{4}{|c|}{ Percentage } \\
\hline & & 1 & 6 & 9 & 4.1 & 2 & $\%$ \\
\hline 2 & $F \quad e \quad v e r$ & 1 & 6 & 9 & 4 & 2 & $\%$ \\
\hline 3 & $\mathrm{Malaise}$ & 7 & & 4 & 1 & 8 & $\%$ \\
\hline 4 & Vom i t i g & 1 & 7 & 1 & 0 & & $\%$ \\
\hline 5 & $\mathrm{n} a \mathrm{u} s \mathrm{e} a$ & 1 & 1 & 6 & 4.7 & 1 & $\%$ \\
\hline 6 & Jointpain & 1 & 1 & 6 & . 7 & 1 & $\%$ \\
\hline 7 & Discomfort & 1 & & 5 & 8 & 8 & $\%$ \\
\hline 8 & Weight lost & 1 & & 5 & 8 & 8 & $\%$ \\
\hline 9 & Loss of appetite & 7 & & 4 & .1 & 8 & $\%$ \\
\hline 10 & Dizziness & 6 & & 3 & . 2 & 9 & $\%$ \\
\hline 11 & Restlessness & 2 & & 1 & 7 & 6 & $\%$ \\
\hline 12 & Anorexia & 8 & & 4 & 0 & 6 & $\%$ \\
\hline 13 & Chills and rigors & 6 & & 3 & 2 & 9 & $\%$ \\
\hline 14 & Body pain & 5 & & 2 & . 4 & 1 & $\%$ \\
\hline 15 & Arthralfia & 1 & & 5 & 8 & 8 & $\%$ \\
\hline 16 & Ble ed i ng & 1 & & 5 & . 8 & 8 & $\%$ \\
\hline 17 & Convulsion & 4 & & 2 & 3.5 & 3 & $\%$ \\
\hline 18 & Insomnia & 3 & & 1 & . 6 & 5 & $\%$ \\
\hline 19 & F a t igue & 1 & & 5 & 8 & 8 & $\%$ \\
\hline 20 & $P$ yrexi a & 1 & & 5 & 8 & 8 & $\%$ \\
\hline 21 & Weakness & 6 & & 3 & 2 & 9 & $\%$ \\
\hline 22 & C $\quad 0 \quad u \quad g \quad h$ & 1 & & 5 & 8 & 8 & $\%$ \\
\hline 23 & Reduce urine output & 1 & & 5 & 8 & 8 & $\%$ \\
\hline 24 & Diarrea & 7 & & 4 & . 1 & 8 & $\%$ \\
\hline 25 & Loss of consciousness & 2 & & 1 & 7 & 6 & $\%$ \\
\hline 26 & Abdominal pain & 5 & & 2 & 4 & 1 & $\%$ \\
\hline 27 & High temperature & 4 & & 2 & 5 & 3 & $\%$ \\
\hline 28 & $\mathrm{~B}$ ack ache & 1 & & 5 & 8 & 8 & $\%$ \\
\hline 29 & Bitter taste & 3 & & 1 & . 6 & 5 & $\%$ \\
\hline 30 & Lips rashes/fever blister & 3 & & 1 & 6 & 5 & $\%$ \\
\hline 31 & Jaundice & 2 & & 1 & 1. & 6 & $\%$ \\
\hline \multicolumn{3}{|c|}{ FOR SYMPTOMS } & \multicolumn{5}{|c|}{$=160$} \\
\hline \multicolumn{3}{|c|}{ Mean for No. of symptoms } & $=$ & \multicolumn{4}{|c|}{5.16} \\
\hline \multicolumn{3}{|c|}{ Deviation from mean for no. of symptoms } & $=$ & \multicolumn{4}{|c|}{$\underline{4.712}$} \\
\hline
\end{tabular}

Table 1 indicates vomiting as the most occurring symptom with $100 \%$, headache and fever with $94.12 \% \mathrm{~m}$ nausea and joint paint with $64.71 \%$, malaise, loss of appetite and diarrhea with $41.18 \%$, dizziness, chills and rigor and weakness with $35.29 \%$, body pain and abdominal pain with $29.41 \%$, convulsion and high temperature with $23.53 \%$, insomnia, bitter taste and lips rashes/fever blister with $17.65 \%$, restlessness, lost of consciousness and jaundice with $11.76 \%$, discomfort, weight loss, arthralfia, bleeding, fatigue, pyrexia, cough, reduced urine output and backache with $5.88 \%$.

Table 2: Gender based Prevalence of Malaria Infection in Male and Female Patients

\begin{tabular}{|l|lrr|l|}
\hline G e n d e r & \multicolumn{2}{|c|}{ No. of Patient Infected } & Infected \% \\
\hline M a l e & 1 & 1 & 9 & $43.91 \%$ \\
\hline F e m a l e & 1 & 5 & 2 & $56.09 \%$ \\
\hline T o t a I & 2 & 7 & 1 & - \\
\hline
\end{tabular}

Table 2 shows prevalence of infection among sexes. 152 females were infected with prevalence of $56.09 \%$ while males 119 were infected with a prevalence of $43.91 \%$. 
Table 3: Prevalence of Malaria Infection

from January to December 2011

\begin{tabular}{|c|c|c|}
\hline Mont $h s$ & No, of Patient Infected & Infected \% \\
\hline Ja n u a ry & $2 \quad 0$ & $7.38 \%$ \\
\hline $\mathrm{February}$ & 1 & $43 \%$ \\
\hline 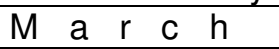 & 1 & $3.69 \%$ \\
\hline $\begin{array}{lllll}A & p & r & i & l\end{array}$ & 2 & $10.70 \%$ \\
\hline M $\quad$ a $\quad y$ & 3 & $11.07 \%$ \\
\hline $\begin{array}{lll}J & u & n \\
\end{array}$ & 3 & $14.02 \%$ \\
\hline $\begin{array}{llll}J & u & \text { l } & y\end{array}$ & 2 & $9.23 \%$ \\
\hline$A \cup g u s t$ & 1 & $5.54 \%$ \\
\hline September & 4 & $15.58 \%$ \\
\hline October & 2 & $10.70 \%$ \\
\hline November & 6 & $2.21 \%$ \\
\hline $\mathrm{Dec}$ e $\mathrm{mb}$ e $\mathrm{r}$ & 1 & $5.54 \%$ \\
\hline
\end{tabular}

Total 271

Mean per month $\quad 22.58$

Table 3 shows the prevalence in the months of the year (201I), which indicates that the highest level of infection occurred in September with $15.58 \%$ prevalence, June with $14.02 \%$, May with $11.07 \%$, April and October with
$10.70 \%$, July with $9.23 \%$, January with $7.38 \%$, august and December with $5.54 \%$, February with $4.43 \%$, March with $3.69 \%$ and November with $2.21 \%$ of prevalence.

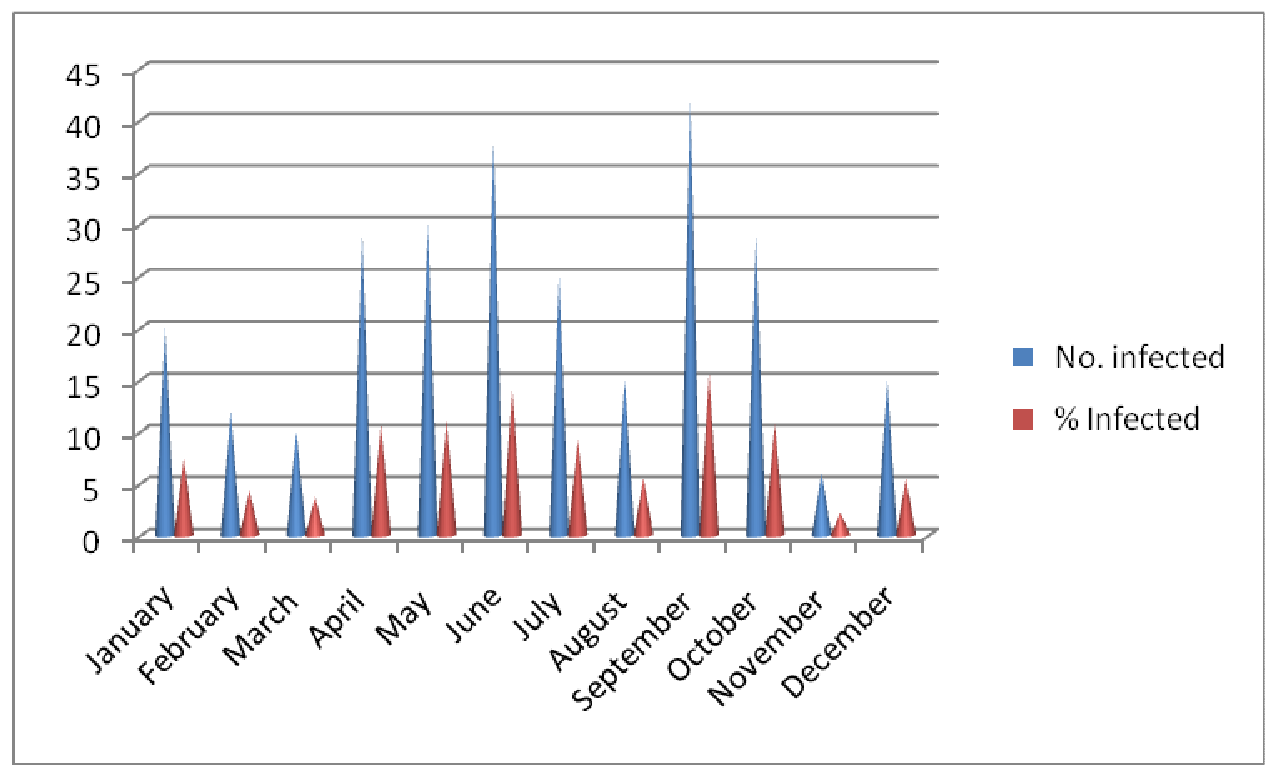

Figure 1: Monthly distribution of malaria prevalence in the study area 


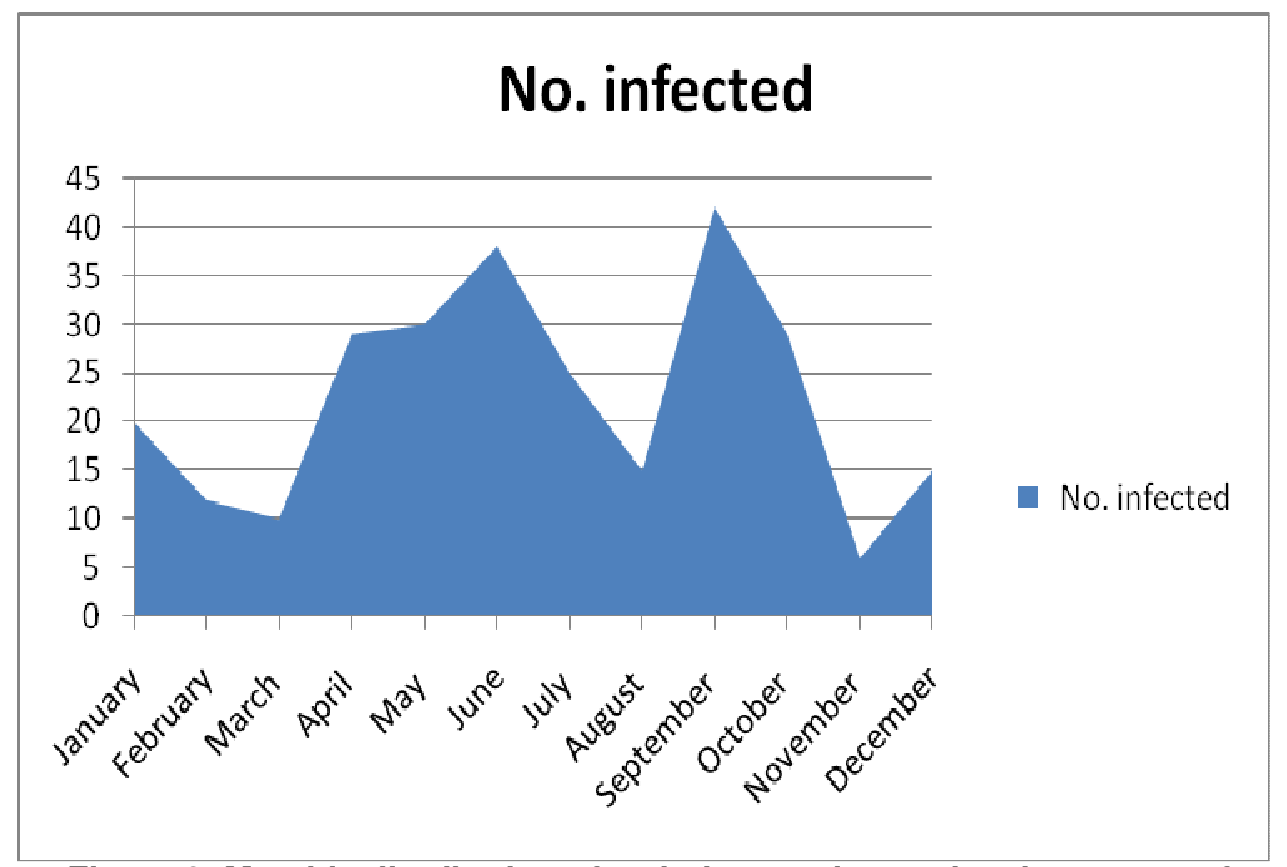

Figure 2: Monthly distribution of malaria prevalence showing nature of skewness within the rainy season

\section{DISCUSSION}

Data on nature and occurrence frequency of symptoms as observed by the medical doctors and nurses was retrieved and used to determine the occurrence frequency of each of the recorded symptoms over the one year period between January, 2011 and December, 2011. From the retrieved data on the medical diagnostic tests results conducted within same period, the monthly prevalence of malaria was evaluated, and the gender based malaria prevalence values calculated with respect to sex of these patients.

The prominently recorded symptoms in these patients included vomiting (100\%), headache/fever (both $94.1 \%$ ), nausea/joint pain (both 64.7\%), anorexia (47.1\%), malaise/loss of appetite/diarrhoea (all $41.2 \%$ ).

Normally, the erythrocytic stage of Plasmodium malaria parasite in the blood involves pathology-related schizogonic activities encompassing bursts of schizonts to release numerous merozoites into the blood stream, with accompanied release of toxic substances (Ukoli, 1990) and reactive oxygen species (ROS) (DescampsLatscha et al, 1987; Postma et al, 1996), which are products of metabolic events within each schizont into the blood stream, increased stress on cells (some of which have damaging effects), alongside some specific cytokines to which capacity to cause damages to body tissues have been clearly associated. ROS such as superoxides anion and the hydroxyl radical are produced by activated neutrophils in the host and during degradation of hemoglobin in the parasite (Ozurumba and Ogundiniyi, 2011; Ozurumba, 2012a). The effects of ROS can be both beneficial and pathological, depending on the amount and place of production which are involved in pathological changes to host tissues such as damage to vascular endothelial lining during malaria infection (Postma et al, 1996). Cytokines (depending on which class) play an important role in ROS related pathology of malaria and this concept and its related studies may bring up new treatment concepts and associated developed drugs (Burke, 2015) and as elucidated in the cytokine and ROS related work of Ozurumba (2012a). These events are prominently responsible for the triggering of emergence of a myriad of symptoms and negative effects on infected persons. This is exemplified in observations involving pains, weakness, tiredness, fever and discomforts it inflicts on such patients. The symptoms of malaria typically develop within 10days to 4weeks following infection and this period varies in infected individuals.

Common causes of vomiting and nausea which may occur separately or together include nature of chemotherapy/medications, gastroparesis (a condition in which the muscle of the stomach wall does not function properly, interfering with digestion), some kind of infections, and intestinal obstruction (Mayo Foundation Clinic, 2016). In the early stages of malaria, the symptoms may include fever, chills, nausea, vomiting, headache, sweats and fatigue, while non-common symptoms include dry cough, enlarged spleen, muscle or back pain or both and in rare cases can lead to impaired functions of brain and spinal cord, and seizures of loss of consciousness (WebMD, 2014).

Stoppler, (2015) reported that vomiting alongside nausea is a symptom of an underlining disease. "Emesis" is the medical term for "vomiting" and added that vomiting can be triggered by a food-borne illness, systemic disease or infections or a result of side 
effect of medication. These are good explanations for the results recorded in this study with high occurrence and presence of prominent symptoms of vomiting, fever, headache, joint pain and anorexia recorded in this study (Table 1). Notably, from the observations in this study, vomiting featured most prominently among symptoms. Vomiting in malaria involves "throwing-up of contents of the stomach" by the patients. In addition, the triggers to these symptomatic observations and events includes the various materials shed into the blood stream during burst of the schizonts, as many of these substances tilt the physiological and homeostatic balance of salts, minerals and fluids in the body and these substances are circulated to various organs in the body of which includes the tongue (which may be coated by these substances or their by-products). This is likely, partly responsible for the involuntary action of throwingup (vomiting) by most of these patients.

Thus, it is suggested that part of the strategies in the treatment of these patients and those in the study area who could be infected and afflicted by malaria should be to include drugs with capacity to help improve the body's homeostatic fluid and substances levels, neutralize toxic effluents from schizogonic bursts which are part of the parasite's life cycle and improve functionality of the taste buds. Vomiting is known to deflate the already ingested food meant to help release energy via cellular respiration from digested carbohydrates, help replace cells which are worn-out functionally inadequate, by effects of these toxic releases into the body (as digested proteins which end up as amino acids aid this cells replacement event). Ingested food lost through vomiting is likely to weaken the body with the accompanied joint pain, nausea and anorexia which were also among the most prominent symptoms recorded (Table 1). It is a known fact that multivitamins or specific vitamins often prescribed by medical doctors also assist patient's recovery from these observed prominent symptoms. These highlighted related counter measures are likely to help in recovery of patients and in containing the rapidly multiplying Plasmodium parasites and the negative effects from their catabolic effluents released into the blood. The positive impact on the individual's state of health and capacity to return to work, profession or trade of such person; thereby saving valuable man hours that contribute to the productive sector of human resources of the economy, impact - on personal and family life, on education and on welfare income (of spouse and children), and contribute to community and regional income through payments of tax and services bills, which are all positive implications that ripple-out on community's socio-economic and health status. These attributes entails the public health connotations. The work of Long et al (2011) gives credence to this discussion.

The gender based prevalence of malaria with respect to sexes (Table 2) was higher for females $(56.1 \%)$ than for males $(43.9 \%)$, though the difference does not look significant. The implication of this result is that both sexes appear to be similarly prone to the risks of infection with Plasmodium malaria parasite in this study population. This observations in areas of gender based malaria prevalence and monthly distribution of the levels of the prevalences is similar to the outcome of the study by Gellert et al (1998) in which the male:female ratio of prevalence of certain symptoms like diarrhea and sickle cell SS disease was 1:5 with the highest prevalence occurring in October (124/480 admissions) and lowest in March (12/303 admissions); and a 1:1.03 male:female ratio in the non-surviving children in the study conducted on children patients of Paedriatics Departmental ward of State Specialist Hospital Maiduguri, Nigeria.

From observations on the monthly occurrence of malaria in the study population, the top levels of prevalences was recorded for the months of September (15.6\%), June (14.0\%), May (11.1\%) and April/October (both 10.1\%) (Table 3). Figure 1 depicts the figures on the monthly prevalences and Figure 2 showed skewness of the peak prevalence values mostly around the months of the rainy season. This connotes a malaria prevalence that appeared seasonal in levels of occurrence for year 2011 (January to December) on which the study was based. However, the study by Umaru and Uyaibasi (2015) revealed that males were more prone to malaria than females in an area in Kaduna State. These authors added that in some areas in Nigeria, malaria exhibits seasonally peaking prevalences for rainy season. They attributed these variants in nature of transmission based on the two major seasons in Nigeria to microclimate, topography, population densities, cultural practices and general way of life which influences malaria intensity and management of the disease. This is in line with the explanations proffered by Ozurumba et al (2006) in their study on malaria using a recombinant peptide R32tet32 as the antigen for determining antibody responses to malaria infection among patients sampled in an area in southwestern Nigeria. Part of the explanations for the seasonal trend in this study can be attributed to the fact that these rainy season months are likely to be well associated with the peak provisions of suitable breeding conditions for the mosquito vector.

\section{CONCLUSION}

This study showed that certain symptoms such as vomiting, fever, headache, joint pain and nausea observed in this study population) appear to be the most prominent malaria symptoms recorded while symptoms such as weight loss, bleeding, reduced uterine output where the least prominent in occurrence. This is possibly because the later symptoms are usually associated with effects of sustained and not well treated/untreated malaria cases in which the parasites are flourishing in multiplicity and are ferried to other vital and sensitive organs of the body such as the brain (for cerebral 
malaria) and the kidney (in which damages to the tissues of the kidney cause physiological and systemic malfunctioning of these organs) and Jaundice (which may be associated with damages to the liver and its related dysfunction).

The sex of the patients was not a predisposing or determining factor for being prone to malaria infection among individuals in this study population, while there appeared to be a seasonally aligned rise in level of malaria prevalence over the months of the year. The pathological and public health implications of our results have been adequately discussed.

\section{ACKNOWLEDGEMENTS}

We authors to this study, thank the Doctors, Nurses and laboratory personnel of UMTH Maiduguri, who released of the laboratory test results we analyzed and provided information through questionnaires from data on patients which we retrieved in our data collection. Ozurumba L.N.- humbly and heartily expresses his gratitude to Walden University Minneapolis USA, for his academic affiliation with the School of Public Health of this Institution.

\section{REFERENCES}

AMCA (2014). Mosquito-Borne Diseases. American mosquito control association $\AA$ Mount Laurel NJ, USA. www.mosquito.org/mosquito-borne-diseases. (March 11, 2016).

Arora, DR and Arora BB. 2010. Medical Parasitology, 3rd Edition. CBS Publishers and Distributors Pvt. Ltd. 227pp.

Burke D. 2015. Malaria Symptoms. Healthline. Reviewed by Lafalame M.R. www.healthline.com/healthline. Retrieved 21st February, 2016.

Cheesbrough M. 1998. District Laboratory Practice in Tropical Countries. Pages 241, 245.

Descamps-Latscha B, Lunel-Fabiani F, Kara-Banis A and Druile P. 1987. Generation of reactive oxygen species in whole blood of patients with acute falciparum malaria. Parasite Immunology. Vol. 9(2): 275-279.

Hanson SM and Craig GB Jr. 1995. Aedes albopictus (Diptera: Culcidae) Eggs: Field survivorship during northern Indiana winter. J. Med. Entomol. 32:599604.

Longo DL, Fausi AS, Kasper DL, Hauser SL, Jameson $\mathrm{JL}$ and Loscalzo J. (Editors). 2011. Harrison's Principles of Internal Medicine, Volumes 1 and 2. $18^{\text {th }}$ Ed. United Sates: McGraw-Hill Professional Global Education Holdings LLC.

LSM. 2016. Malaria Control Program, Lagos State ministry of health. www.lagosstateministryofhealth.com/programme_inf o.php. Retrieved $11^{\text {th }}$ March, 2016.

Mayo Foundation. 2016. Mayo Clinic: Nausea and Vomiting. By a Mayo staff, USA. (c) 1998-2016. www.mayoclinic.org/symptoms. (February 24, 2016).

Noland GS, Graves PM, Sallau A, Eigege A, Emukah E and Patterson AE. 2014. Malaria prevalence anemia and baseline intervention coverage prior to mass net distribution in Abia and Plateau States, Nigeria. BMC Infectious Diseases, March, 14: 168.

Oaks SC, Mitchell VS, Pearson GW and Carpenter CJ. (eds). 1999. Malaria: Obstacles and Opportunities. National Academy Press. Washington DC: 13-24: 122-123.

Ozurumba LN. 2012a. A tentative flow chart for the breakdown of haemoglobin by Plasmodium, haemozoin formation, potential therapeutic targets and effect of chemotherapy $\{A$ review $\}$. Adamawa State University Jnl of Scientific Research. Vol. 2 (1): 115 - 122. $h t t p / / w w w . a d s u . e d u . n g$.

Ozurumba LN and Ogundiniyi OS. 2011. Dynamics of host- parasite immune response involving malaria pigment containing leucocytes: a descriptive case 40 study. International Journal of Applied. Biological. Research. Vol. 3 (1): 26 - 38. http//www.ijabr.com.

Ozurumba LN, Anumudu $\mathrm{Cl}$ and Nwagwu M. 2006. Seasonal variations in antibody response to a Plasmodium falciparum recombinant circumsporozoite antigen in two villages in south western Nigeria. African Journal of Biomedical

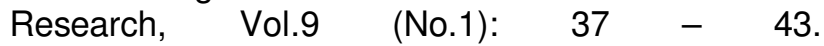
http://www.bioline.br/md.

Postma NS, Zuidema J, Mommers EC and Eling WMC. 1996. Oxidative stress in malaria; implications for prevention and therapy.

Romi R, Severini F and Toma L. 2006. Cold acclimation and overwintering of female Aedes albopictus in Roma. Journal of the American mosquito control association 22 (1): 149-151.

Stopper MC. (MD). 2016. Vomiting symptoms and signs. Reviewed by a medical Doctor in2015. www.medicinenet.com. (February 22, 2016).

Udon EE, Oyo-Ita AE, Odey FA, Eyong KI, Oringanje M, Oduwole O.A. et al (2013). Malariometric indices among Nigerian Children in a rural setting. Malaria Research and Treatment, 716805, Feb 2013.

Ukoli, FMA. 1990. Introduction to Tropical Parasitology in Tropical Africa. Textflow Ltd., Ibadan, Nigeria. 464pp.

Umaru ML and Uyaibasi GB. 2015. Prevalence of malaria in patients attending the General Hospital Makarfi, Kaduna State, North America, Journal of Infectious Diseases and Microbiology, 3(1):1-5.

WebMD. 2016. Healthwise- Health concern on your mind. WebMD Medical Reference from Healthwise. Last updated June $4^{\text {th }}, 2014$. WebMD LLC. (C) 20052016. (February 21, 2016).

WHO. 2013. WHO Report 2013, WHO Geneva, Switzerland, Dec 2013. (March 11, 2016). 
WHO. 2016a. WHO 10 Facts on malaria. WHO. 2016b. Fact sheets on malaria. www.int.features/factfiles/malaria/en. (March 11, www.who.mediacentre/factssheets/. (March 11, 2016). 2016).

Cite this Article: David J, Anaso EC, Ozurumba LN (2016). Prevalence of Malaria in a Study Population in Maiduguri, Nigeria, Symptomatic Manifestations and Public Health Implications. Greener Journal of Biological Sciences, 6(5):103-111, http://doi.org/10.15580/GJBS.2016.5.092416145. 